\title{
POLÍTICAS, ENSEÑANZA Y APRENDIZAJE DE LAESCRITURA EN LA UNIVERSIDAD
}

\section{POLICIES, TEACHING AND LEARNING OF WRITING IN THE UNIVERSITY}

\author{
Azael Correa Carvajal' \\ Naryi Lorena Caro Sotelo² \\ Facultad de Ciencias de la Educación. \\ Universidad de la Amazonía \\ Florencia, Caquetá, Colombia
}

\section{RESUMEN}

Este artículo presenta los resultados más relevantes de la investigación Prácticas de la enseñanza y aprendizaje de la escritura, que se realizó con el propósito de comprender las prácticas de enseñanza y aprendizaje de la escritura en el ciclo básico de la Facultad de Ciencias de la Educación en la Universidad de la Amazonia. La metodología empleada fue de análisis mixto, es decir, tanto cualitativa como cuantitativa, usando como técnicas la revisión

1 Docente Universidad de la Amazonia. Doctorando en Humanidades, Humanismo y Persona. Énfasis en Antropología de la Educación (Universidad San Buenaventura - Bogotá). Magíster en Enseñanza de las Ciencias. Especialista en Pedagogía. Licenciado en Lengua Castellana y Literatura. E-mail: azael.correa@hotmail.com CVLAC: https://scienti.minciencias.gov.co/cvlac/visualizador/generarCurriculoCv.do?cod_rh=0000009662 Teléfono: 3208482484 2 Estudiante de Psicología. Universidad de la Amazonía. E-mail: n.caro@udla.edu.co ORCID: https://orcid. org/0000-0002-4211-2225 Teléfono: 3212808199 documental, entrevistas y encuestas. Los resultados permitieron comprender que existen algunas falencias entre las actuales políticas de la escritura, sus procesos de enseñanza y aprendizaje, como también que los procesos de enseñanza de la escritura siguen siendo tradicionales y los estudiantes en la mayoría de los casos escriben por obligación. A la vez postular la necesidad de poner a dialogar coherentemente las políticas de la escritura y sus prácticas de enseñanza y aprendizaje en la universidad.

PALABRAS CLAVE: Prácticas, escritura, política, enseñanza y aprendizaje.

\section{ABSTRACT}

This article presents the most relevant results of the research Practices of teaching and learning of writing, which was carried out with the purpose of understanding the teaching and 
learning practices of writing in the basic cycle of the Faculty of Sciences of the Education at the University of the Amazon. The methodology used was a mixed analysis, that is, both qualitative and quantitative, using documentary review, interviews and surveys as techniques. The results allowed us to understand that there are some shortcomings between the current policies of writing, their teaching and learning processes, as well as that the teaching processes of writing are still traditional and students in most cases write out of obligation. At the same time, postulate the need for a coherent dialogue on writing policies and their teaching and learning practices at the university.

KEYWORDS: Practice, writing, politics, teaching and learning.

\section{INTRODUCCIÓN}

La escritura representa uno de los mayores retos en la educación superior, puesto que, al ser un pilar fundamental para la construcción de conocimiento, implica poner en coherencia tanto sus políticas como sus prácticas de enseñanza y aprendizaje. Precisamente con el propósito de conocer el estado actual de las políticas y de las prácticas de la enseñanza y el aprendizaje de la escritura - puesto que sobre la misma se especula mucho - en el marco del semillero Aletheia adscrito al Programa de Lengua Castellana y Literatura de la Facultad de Ciencias de la Educación, se puso en marcha la investigación Prácticas de la enseñanza y aprendizaje de la escritura en el ciclo básico de la Facultad de Ciencias de la Educación en la Universidad de la Amazonia.

Por ser referentes sobre este campo del saber, se consideró pertinente asumir las posturas teóricas de algunos autores entre los que se encuentran Pérez y Rincón (2013), Carlino (2007), Vargas (2005) y Cassany (1993). Estos autores insinúan que la enseñanza y el aprendizaje de la escritura son procesos que si bien tienen marcas sociales - la cultura escritora del mundo social y escolar determina en gran medida los posteriores procesos de aprendizaje - deben ser asumidos en plenitud por el escenario académico universitario, en ese sentido, parte de la labor de la universidad consiste en ayudarles a los estudiantes a superar las limitaciones de aprendizaje con las que llegan a la educación superior y a través de la escritura posibilitarles su inserción a sus nuevas culturas disciplinares del saber.

Para abordar esta investigación se definieron 3 categorías centrales: la primera Política de la escritura, la segunda Enseñanza de la escritura y la tercera Aprendizaje de la escritura. Se pudo evidenciar que existen serias rupturas entre las políticas de enseñanza y aprendizaje de la escritura y sus prácticas de enseñanza y aprendizaje, lo que de alguna manera se podría considerar también como el mayor aporte de esta investigación y que a la vez se convierte como referente para considerar un replanteamiento de las actuales políticas de enseñanza y aprendizaje de la escritura y del mismo modo sus prácticas de enseñanza y aprendizaje.

\section{MARCO TEÓRICO}

\section{POLÍTICA}

Las políticas son directrices que ayudan a orientar un proceso con el fin de conseguir un objetivo, son principios que se deben seguir para ordenar, facilitar y direccionar lo que una determinada comunidad desea para su bien; según Fragoso (2006), en la estructuración de las políticas se deben establecer acuerdos con otras personas - actitud política - que, a su vez conduzca a la organización del grupo social.

Una de las áreas más importantes de la vida social tiene que ver con la educación. Tomando las palabras de Fragoso, la misma educación no puede prescindir de ciertas políticas o directrices para su estructuración, desarrollo y evaluación. 
De esta forma, cada uno de sus procesos implícitos, por ejemplo, la lectura, la evaluación $\mathrm{y}$, por supuesto, la escritura - problemática con antecedentes socioculturales - (González Pinzón et al., 2015), debería estar orientada por unas ciertas políticas.

Universidades como las de Australia, hace una década y media apoyan los "Centros de Escritura y Aprendizaje", con el objeto de fortalecer el proceso escritural. Dentro de las políticas para su promoción y fortalecimiento se encuentran estatutos, recursos, formación de sus docentes y organización periódica de foros académicos sobre el tema (Carlino, 2009). Lo que, de alguna forma, genera un estado de alarma sobre el estado de las políticas de la escritura en otros países que no son abanderados en los procesos escriturales.

Las políticas dentro del ejercicio de la escritura, deben ser reconocidas como parte fundamental del bienestar social y de la calidad de vida colectiva, como lo plantea Barbero y Lluch (2011), pensar en las prácticas lectoescritoras como método para la inclusión y la cohesión social es una manera de que la ciudadanía participe. Así mismo lo afirman Cassany y Morales (2009) quienes plantean que la lectura y escritura no solo son herramientas fundamentales para lograr una adecuada práctica profesional y científica, sino que también sirven para que estas personas sean activas y participativas en sus comunidades.

En Colombia una de las primeras universidades que se interesó en ubicar la lectura y escritura como parte importante en el terreno académico fue la Universidad Nacional de Colombia, primero con el curso "Español Instrumental" desde el año 1984 de la Facultad Ciencias Humanas y otro curso llamado "Comprensión y Producción de Textos" de la Facultad de Medicina creado a finales de la década de los 80. Estos cursos iniciaron con una perspectiva formal de la lengua referente a la estructura, es decir, a reglas gramaticales y morfosintácticas.

Estas brindaban herramientas básicas en la producción y comprensión en general, no tenían contenido específico ni producción de textos académicos o científicos. Luego en el año 1994 se promulgó la ley 115 la cual replanteó entre otros aspectos el rol que jugaban la lectura y la escritura en los niveles de formación básica y media. En 1996 se creó Cátedra UNESCO para la lectoescritura bajo una tutela de la Universidad del Valle, cuyo fin fue contribuir a mejorar la calidad de la educación en América Latina (González y Vega, 2014).

En este sentido, la creación y el respaldo a los lineamientos que orienten las prácticas de escritura no está de más, sino que contribuye en muchos aspectos a la formación universitaria. Parte de las bondades de las políticas escriturales, su enseñanza y aprendizaje, es que ofrece - sin ser camisa de fuerza mayor - unos determinados referentes teórico conceptuales, ciertas normas y directrices para escribir, aligera las cargas, facilita la planeación y la ejecución del trabajo intelectual y posibilita un uso pertinente del tiempo, etc. (Patiño, 2013).

\section{ENSEÑANZA}

La enseñanza es un proceso de instrucción a través del cual se transmiten conocimientos previos que edifican nuevos saberes. Por lo menos se podrían encontrar dos modelos de enseñanza. El primero de ellos puede denominarse como enseñanza bancaria, es decir, centrada en la transmisión de información; según Freire (2011), este modelo en el cual cuyo sujeto central es el docente, conduce a los estudiantes a la memorización mecánica de los contenidos. Los estudiantes se transforman en "vasijas", en recipientes que deben ser "Ilenados" por los docentes. Entre más información reciban los estudiantes significa que son mejores estudiantes. 
Sin embargo, actualmente en la perspectiva de un nuevo horizonte pedagógico se puede hablar de un segundo modelo de enseñanza, el basado en competencias. El estudiante es el gestor de su propio conocimiento, a través de diversas actividades que le ayuden a acrecentar sus saberes por medio del aprendizaje autónomo, además se apoya en la motivación y el desarrollo de habilidades intelectuales superiores (Sesento, 2008). En este proceso el docente es una guía, ya que orienta el aprendizaje sin imponer información a sus estudiantes, generando en ellos un aparente pensamiento competitivo y crítico.

Un aspecto fundamental en la enseñanza como quehacer diario de los docentes es la escritura en cuanto proceso superior de la mente que posibilita la construcción de identidad académica. Según Zhizhko (2014) la escritura es un sistema de representación gráfica de una lengua, que se manifiesta por medio de signos, y se convierte en un medio de comunicación entre las personas. Pero no solo de comunicación, sino como ya se advirtió, de subjetivación humana.

Esta comporta un fuerte potencial epistémico. Si de enseñanza se trata, la escritura permite una interacción entre docentes y estudiantes, con el fin de concretar entre ambos la certeza de las frases, la coherencia y los conceptos, entre otros; esta relación ayuda a fortalecer el entendimiento de las temáticas (Giraldo, 2015). Por ello en la universidad, su enseñanza debe ser un eje transversal. Parte del reto está en acompañar los procesos de planeación, redacción, revisión y cualificación de la escritura dentro y fuera del aula (Mendoza, 2016).

Los docentes cumplen una función elemental en los procesos de motivación en los estudiantes, fomentar el uso de la escritura tiene implicaciones académicas muy amplias, ya que puede producir efectos positivos en el rendimiento de los estudiantes. Graham, Harris y Larsen (2001) señalan la importancia de la motivación como medio para enseñar los procesos escritos, creando un ambiente adecuado y cómodo para escribir, incentivando el interés de los estudiantes con los temas, especificando los objetivos de los procesos, promoviendo la autoeficacia y modelando con actitudes positivas a través de herramientas que ayuden a mejorar su comportamiento en clase y en su vida cotidiana.

Resulta trascendental fortalecer las prácticas de escritura en su función epistémica, de modo que se hallen las capacidades de todos los estudiantes (Pérez y Rincón, 2013). Pero no solo la epistémica. Enseñar la escritura es un proceso determinante y útil, tanto así que, para muchos, la escritura se ha convertido en una auténtica posibilidad de construcción de identidad académica. La universidad tiene el reto de fortalecer la enseñanza de este proceso y eso implica, entre otras cosas, cualificar la formación docente.

\section{APRENDIZAJE}

El aprendizaje se ha definido como el proceso por el cual se adquieren o se transforman ideas, habilidades y capacidades a través de técnicas (Zapata-Ros, 2015). Este proceso implica el desarrollo mental y la disposición de cada ser humano. Concha, García, y Fonseca (2015) manifiestan que las estrategias de aprendizaje son de gran importancia para desarrollar capacidades como la resolución de problemas, el aprendizaje autónomo y trabajo en equipo.

En cuanto a la escritura, su aprendizaje, como lo plantea Cassany (1996), ha sido un proceso que ha generado un sinnúmero de sistemas alfabéticos para la comunicación humana, además generan y administran información y conocimiento. Escribir es un medio para aprender y construir el mundo, no es una simple destreza, es una posibilidad de crecimiento continuo ya que su práctica además de servir para conocer o dar a conocer, también reforma 
la conciencia (Giraldo, 2015), de ahí que su aprendizaje no debe ser visto como una forma de transmitir datos, sino transversal a las disciplinas humanas.

En la universidad, aprender sobre sobre escritura debe ser visto como un eje fundamental para el desarrollo de las prácticas educativas, sin embargo, en muchas ocasiones se centra en la transmisión de información y no en que se consolide el conocimiento. Vale la pena recordar a Zuluaga sobre este asunto. "La escuela pierde su norte cuando se preocupa más por la enseñanza que por el aprendizaje (...) y es evaluada más por sus efectos cuantitativos, estadísticos, pero no desde la esencia de su pedagogía" (2008).

El alumno universitario debe entender que el aprender a escribir requiere de tiempo y dedicación. Vargas (2005) hace una afirmación importante al respecto: escribir es un proceso que se debe realizar desde la planeación seguido de la revisión y la corrección constante lo que le va a permitir a los alumnos mejorar con su práctica, por ello es importante que el estudiante asuma la responsabilidad de su educación autónomamente, de manera consciente del proceso, orientado con las herramientas que les dan los docentes para que sea de manera eficaz.

El proceso escritural trae muchos beneficios para el bienestar psicológico de los estudiantes y, la adquisición de competencias individuales y sociales que alimentan la capacidad para crecer, tanto en el ámbito educativo como en el personal. Colmenares y Delgado (2008) expresan al respecto que a medida que se crece académica y personalmente, el estudiante adquiere destrezas que lo ayudan a solucionar problemas, presto a la solución de las situaciones académicamente difíciles, lo que genera cada vez una construcción integral más compleja sobre la realidad social e individual.
Según Uribe y Camargo (2011), existen varios tipos de estrategias que son utilizadas por los estudiantes y promovidas por los docentes para mejorar su aprendizaje, entre las más usadas se encuentra el uso de apuntes para repasos, uso del diccionario para consultar palabras que generen confusión, poseer un propósito claro, identificar ideas principales de los textos y al finalizar realizar una evaluación.

En últimas, es importante mencionar que ninguno de los 3 conceptos abordados (políticas, enseñanza y aprendizaje), deben ser tomados de manera aislada. Por lo contrario, lo que esta investigación pretende demostrar es que, la educación y, por supuesto, la misma escritura universitaria demanda poner en orden $y$, por lo tanto, en coherencia, sus políticas de enseñanza y aprendizaje. Pocas utilidades tienen unas políticas sobre la escritura, si estas no son revisadas, evaluadas, cualificadas o si los docentes las desconocen, no orientan sus prácticas a través de estas y, en el peor de los casos, el aprendizaje aparece divorciado de las mismas.

\section{METODOLOGÍA}

Para el desarrollo de la investigación se planteó una metodología de análisis mixto, ya que los datos cuantitativos permitieron trascender hacia un análisis cualitativo de la investigación, de tal forma que la triangulación de la información a partir de ambos métodos permitió encontrar varios caminos para comprender e interpretar los datos (Guelmes y Nieto, 2015). La ruta metodológica de la investigación estuvo estructurada en un primer momento mediante el planteamiento de las categorías centrales de la investigación, a saber, Política de la escritura, la Enseñanza de la escritura y Aprendizaje de la escritura, en un segundo momento realizando el trabajo de campo a través de las técnicas revisión documental, entrevistas y encuestas, y tercero la triangulación de la información y análisis de la misma. 
Considerando que el propósito central de la investigación era comprender el estado de las políticas y de las prácticas de la escritura se plantearon las categorías centrales de la investigación y las técnicas de investigación. De esta manera para lograr comprender el estado de las políticas de la escritura se propuso la técnica revisión documental, para comprender el estado de las prácticas de enseñanza de la escritura se propuso la técnica entrevista y para comprender el estado de las prácticas de aprendizaje de la escritura se propuso la técnica encuesta.

Para la recolección de información en primer lugar se identificaron los semestres que conforman el ciclo básico de cada carrera de la Facultad de Ciencias de la Educación, proyectando una muestra con un cálculo del intervalo de confianza de por lo menos $90 \%$ de fiabilidad. Las carreras que conforman la muestra de estudio fueron Psicología, Lengua castellana, Licenciatura en Educación artística y cultural, Licenciatura En Educación Física, Recreación y Deporte y Licenciatura en Ciencias Sociales y el espacio académico seleccionado fue Comunicación en su nivel básico y avanzado puesto que desde estos espacios se movilizan ciertos procesos aprendizaje de la escritura.

En relación con el trabajo de revisión documental se seleccionaron como muestra un total de 92 textos entre los que se encuentran los documentos rectores de los programas muestra, los planes de trabajo y los acuerdos pedagógicos realizados entre los docentes que orientaban el espacio académico de Comunicación en sus dos niveles y los estudiantes participantes de los mismos. Del mismo modo se realizaron a los docentes 40 entrevistas compuestas por un cuestionario de 16 preguntas que fueron grabadas en audio, sumado a esto se efectuaron 151 encuestas conformadas por 7 preguntas para los estudiantes que asistían a las clases anteriormente mencionadas.
Los documentos de política se analizaron a través de una matriz que contemplaba aspectos como enfoque de la enseñanza de la escritura, tipo de prácticas para su enseñanza y aprendizaje, entre otros. Las entrevistas se transcribieron en su totalidad en un documento de Word, posteriormente en un documento en Excel a la luz de aspectos como metodología de la clase, tipo de textos que se promueven escribir, posturas teóricas para su enseñanza, entre otros. La sistematización de las encuestas se organizó en un documento en Excel, considerando aspectos como motivaciones para la escritura, tipo de espacios y de tiempos en los que escribe, entre otros. Finalmente, la sistematización y organización de la información dio paso a la triangulación y análisis de la información de las que se derivaron los resultados y las proposiciones de la investigación.

\section{RESULTADOS DE LA INVESTIGACIÓN}

\section{POLÍTICA}

Las políticas públicas "entendidas como programas de acciones, representan la realización concreta de una serie de decisiones legitimadas por el Estado en su voluntad de modificar la distribución de valores y recursos disponibles mediante la acción sobre el comportamiento de actores sociales" (Roth, 2002). Luego de revisar y analizar una serie de documentos como Programas de Cursos, Acuerdos Pedagógicos, Planes de Estudio, Planes de Evaluación, entre otros, se evidenció que estos documentos privilegian la escritura de: exámenes, informes de lectura, presentaciones, informes de práctica, reseñas, notas de clase, textos audiovisuales, PowerPoint, resúmenes, proyectos de investigación, textos literarios, tesis y talleres; como se aprecia en la Tabla 1. 
Tabla 1: Tipologías textuales utilizadas en las políticas de la Facultad de Educación.

\begin{tabular}{|l|l|c|c|c|c|}
\hline \multicolumn{1}{|c|}{ Tipología textual } & $\begin{array}{c}\text { Lengua } \\
\text { castellana y } \\
\text { literatura }\end{array}$ & Psicología & $\begin{array}{c}\text { Ciencias } \\
\text { sociales }\end{array}$ & $\begin{array}{c}\text { Artística y } \\
\text { cultura }\end{array}$ \\
\hline $\begin{array}{l}\text { Literario (poesía, cuento, } \\
\text { novela. caricatura) }\end{array}$ & 2 & 0 & 0 & 0 \\
\hline & Presentaciones & 3 & 0 & 3 & 4 \\
\hline & PowerPoint & 2 & 0 & 2 & 0 \\
\hline & Ensayo & 1 & 1 & 11 & 3 \\
\hline & Resumen & 0 & 1 & 2 & 0 \\
\hline & Reseña & 1 & 0 & 5 & 2 \\
\hline & Examen & 6 & 6 & 13 & 1 \\
\hline & Nota de clase & 1 & 1 & 1 & 2 \\
\hline & Informe de lectura & 5 & 5 & 3 & 4 \\
\hline & Textos audiovisuales & 0 & 0 & 3 & 1 \\
\hline & Tesis & 0 & 0 & 1 & 0 \\
\hline & Trabajo de grado & 0 & 0 & 0 & 0 \\
\hline & Artículo científico & 0 & 0 & 0 & 0 \\
\hline & Proyecto de Investigación & 0 & 2 & 0 & 0 \\
\hline & Informe de práctica & 0 & 6 & 3 & 0 \\
\hline & Guión & 0 & 0 & 0 & 0 \\
\hline & Texto periodístico & 0 & 0 & 0 & 0 \\
\hline & Historia de vida & 0 & 0 & 0 & 0 \\
\hline & Taller & 0 & 0 & 1 & 0 \\
\hline & & & & \\
\hline
\end{tabular}

En el programa de Lengua Castellana se realizó el análisis de 18 documentos. La tipología textual más promovida son los exámenes, seguida de informes de lectura y presentaciones. En cuanto a los enfoques se mencionan el sociocultural y el literario. La metodología que más propone tiene que ver con las clases magistrales y las exposiciones de los estudiantes. Respecto a los criterios de evaluación, quedan reducidos a la originalidad.

Por su parte, en el programa de psicología se tuvieron en cuenta 17 documentos. Las tipologías que más se presentan son exámenes e informes de práctica, seguida por informes de lectura. En cuanto al enfoque no se explicita en la mayoría de los textos, pero el más nombrado es el cognitivo. Los talleres y las exposiciones se postulan como metodología para la enseñanza de la escritura, tal como se evidencia en la propuesta de desarrollo programa de curso de comunicación del programa "se realizarán en igual forma, talleres para la práctica y el manejo de los temas propuestos". (p. 3). Respecto a los mecanismos de apoyo se resalta la socialización y retroalimentación dentro de los textos y lo que más se propone a evaluar es la claridad en la expresión de las ideas.

En la carrera de Ciencias Sociales se obtuvo el análisis de 29 documentos. Las tipologías textuales más usadas son exámenes, seguido 
de ensayos y reseñas, siendo el programa que más escribe textos de carácter argumentativo. Aunque no especifican la propuesta frente a la escritura, muchos lineamientos nombran un enfoque sociocultural, comunicativo y constructivista, esta última concepción se entiende como "capacidad de aprendizaje, implica el desarrollo de habilidades cognitivas para resolver problemas y atribuir un significado a aquello que es objeto de estudio, basándose en sus conocimientos e incorporando lo aprendido a sus esquemas conceptuales, readaptando y reorganizando su saber" (Baeza, 2012). Por otra parte, las clases magistrales y los talleres se mencionan como parte de la metodología. En cuanto al mecanismo de apoyo se pide usar la socialización y respecto a los criterios de evaluación se tiene en cuenta la originalidad.

En el programa de artística se revisaron 9 documentos. En este programa toman como referente el documento Maestro de la Facultad: "la Institución se proyecta como una organización comprometida en dinamizar procesos conducentes a la generación del conocimiento científico y tecnológico, ... También propende por la difusión y apropiación del conocimiento científico y tecnológico con los sectores académicos". (p.152). Al contrario de las demás carreras, las tipologías textuales más usadas son presentaciones e informes de lectura, seguida de ensayos, reseñas y notas de clase. El enfoque sugerido, no está especificado en muchos textos, no obstante, en algunos se presenta el enfoque comunicativo como opción. La metodología propuesta tiene que ver con las exposiciones y los talleres, además que los mecanismos de apoyo se centran en la socialización y la retroalimentación. No se especifican los criterios de evaluación, pero se usa en ocasiones el nivel de argumentación.

Conviene recordar algunas de las palabras de Flower y Hayes (1996) quienes realizaron valiosos aportes a la psicología cognitiva, expresaron que la tarea de componer textos escritos promueve cambios importantes tanto en lo referido al tópico acerca del cual se escribe como a los elementos lingüísticos. A partir de la investigación se puede evidenciar que se deben fortalecer los documentos sobre las políticas de la escritura, donde se oriente con mejor claridad las metodologías, enfoques, los tipos de texto que se desea construir para crear cultura académica en los estudiantes, lo que permitiría que ellos se construyan como buenos profesionales y la universidad crezca científicamente.

\section{ENSEÑANZA}

El proceso de enseñanza es fundamental para el desarrollo de la escritura y es deber de los maestros desde los primeros años de escolarización instruirla e incentivarla. Es necesario crear estrategias de enseñanza para conseguir un excelente aprendizaje pues "la enseñanza no puede entenderse más que en relación al aprendizaje; y esta realidad relaciona no sólo a los procesos vinculados a enseñar, sino también a aquellos vinculados a aprender" (Meneses, 2007).

Para la investigación, fue importante conocer cuál es el proceso de enseñanza que usan los docentes. Por ello se realizaron 40 entrevistas a los docentes sobre la enseñanza practicada en ese enfoque, esta entrevista se dividió en dos partes, en la primera se indagó sobre las políticas, para saber si conocen algunos documentos orientadores sobre la enseñanza de la escritura; en caso de que estuvieran enterados sobre ellos, debían explicar qué mencionaban estos documentos sobre la enseñanza en relación con: enfoque, método, estrategias, y además si en el acuerdo pedagógico se fijan pautas sobre el aprendizaje de la escritura.

La segunda parte llamada enseñanza en el aula, se interroga a los docentes si existe una materia directamente responsable de la enseñanza de 
la escritura, si conocen sobre alfabetización académica y escritura epistémica, también cómo realizan los procesos de enseñanza y aprendizaje en los diferentes programas donde orientan cátedra, además el enfoque, mecanismos de escritura, criterios de evaluación, tipologías textuales, propósitos y lugares de la escritura, con el fin de conocer de cerca las prácticas más utilizadas por los docentes. Luego de hacer las entrevistas a los docentes, se procedió a sistematizarlas en una matriz, allí se subrayaron los resultados más relevantes, lo que permitió corroborar la información para posteriormente triangularla con las otras partes de la investigación.
A partir de los resultados se puede evidenciar que el $50 \%$ de los docentes entrevistados, no conocen la existencia de documentos que hablen o den directrices sobre la escritura, como se muestra en la Figura 1. Un claro ejemplo es la Licenciatura en Educación Artística y Cultural donde el $83 \%$ de los docentes de esta carrera no saben sobre estos lineamientos, seguida del programa de Lengua Castellana con un $50 \%$. Desde este punto se puede analizar que el proceso de la escritura así esté estructurado en documentos, si no es socializada con los docentes no será aplicada con éxito a los estudiantes. En cuanto a los docentes que dicen sí conocer estos documentos, destacan que estos hablan sobre proyectar la escritura y orientarla por medio de líneas de investigación.

Figura 1: Diagnóstico del conocimiento de docentes acerca de documentos sobre la escritura en la Facultad de Educación.

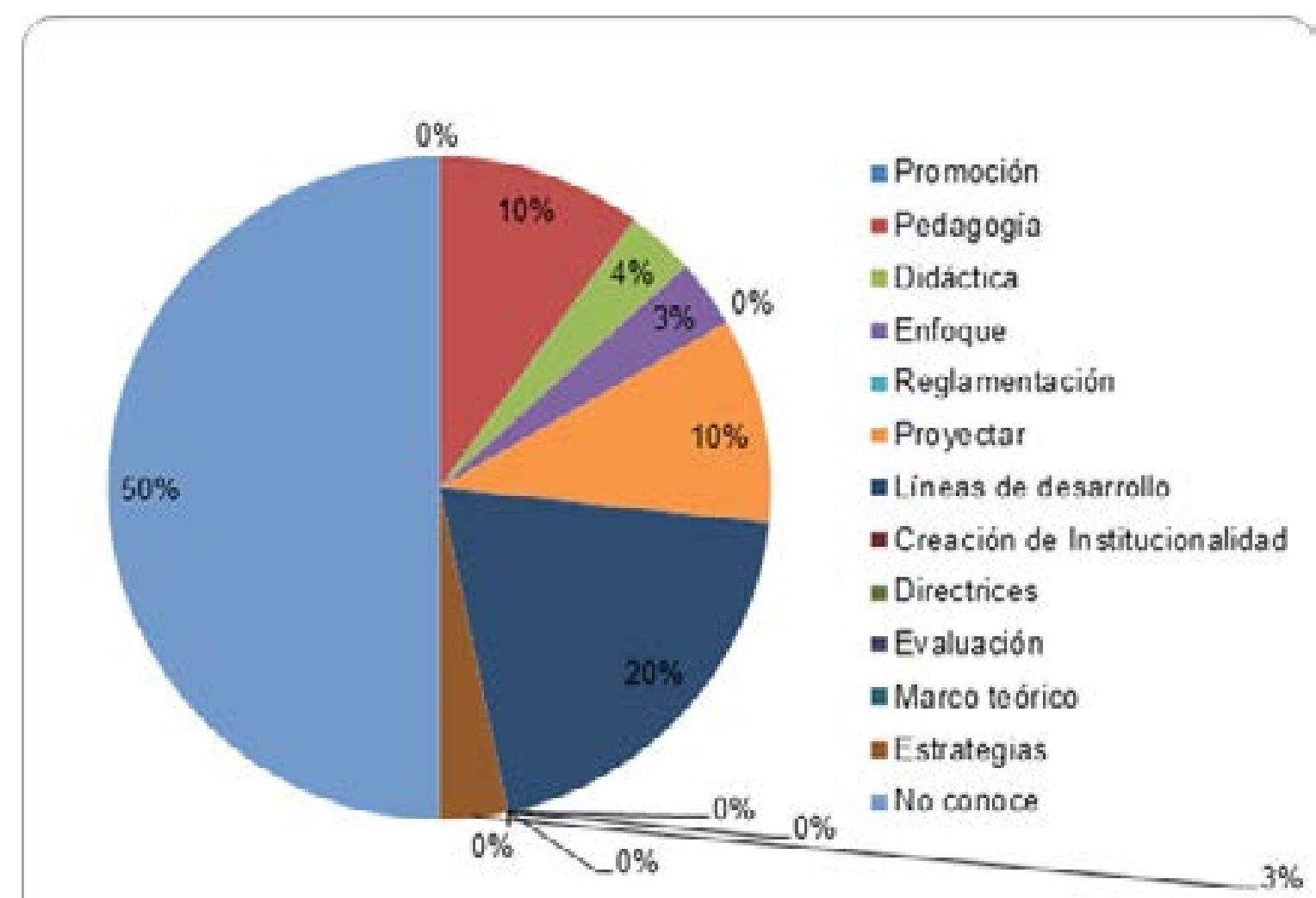


Respecto a los enfoques, aquel que más implementan los docentes es el constructivismo, ya que un $28 \%$ de ellos lo usa, este enfoque permite la participación activa de los estudiantes, sin embargo, otro $28 \%$ dicen utilizar otros, lo que quiere decir que no existe una ruta delimitada sobre los enfoques que se deben usar. En el programa de Sociales es donde más se aplica el método constructivista con un $50 \%$. Los tipos de enfoques menos utilizados a nivel general son el crítico y el comunicativo con un $3 \%$.

La metodología también fue evaluada, esta se entiende como la manera en la cual los docentes desarrollan sus clases. Esto se tuvo en cuenta ya que "es necesario reflexionar sobre la manera de enseñar y aprender, atendiendo a las necesidades del alumnado del siglo XXI y buscando la manera de generar conocimiento y experiencias académicas, profesionales y personales significativas y de calidad." (Villalonga y Nuere, 2017). Un 33\% de los docentes usan como metodología las clases magistrales, seguido de los talleres a estudiantes. Es importante mencionar que, en la carrera de Lengua Castellana el 38\% de los maestros usan las asesorías como metodología.

Los mecanismos de apoyo usados por los docentes indican la manera en que se puede brindar servicios de ayuda para que se logre un mayor aprendizaje de los estudiantes, los resultados arrojados lograron evidenciar que el $28 \%$ de los docentes entrevistados usan la socialización como un método de apoyo, y el $25 \%$ usan la retroalimentación; la carrera que más usa el ultimo método es Licenciatura en Lengua Castellana, con un $67 \%$ de los docentes de la carrera y los de psicología con un $40 \%$. Los docentes de educación física no tienen alguno en específico.

Respecto a las tipologías textuales los resultados pudieron develar que el $33 \%$ de los docentes entrevistados usan ensayos para sus actividades, seguido de informes con un $15 \%$.
La carrera que hace menos uso de los ensayos es artística usando como principal método los informes de lectura con 33\%. Por otra parte, los criterios de evaluación manejados pueden ser sumamente variados, se pudo notar que la mayoría de los docentes tienen en cuenta el nivel de argumentación en los textos exigidos a los estudiantes, seguido de la cohesión y coherencia, donde la carrera en la cual prima este segundo criterio es Lengua Castellana con un $40 \%$ de los docentes.

A la hora de realizar el proceso de escritura se debe tener en cuenta cuál es el propósito de esta, entre las opciones mencionadas por los docentes, el $34 \%$ de estos consideran que es la escritura epistémica, esta tiene como fin escribir para entender y pensar mejor los temas. Asimismo, los lugares en los cuales los docentes hacen que los estudiantes practiquen la escritura son dentro y fuera del aula de clases en un $65 \%$, mientras un $21 \%$ dicen que se realiza solamente a la hora de la clase.

Se puede concluir que la enseñanza de la escritura es un cimiento para que los estudiantes se formen en la academia, al no tener una directriz clara sobre la misma se diluye la intención del aprendizaje. Se puede notar a partir de los resultados que no existe un conocimiento claro de las políticas que deben orientar este proceso, es decir cada docente elige la manera, el momento y el tema en los cuales sus estudiantes deben escribir. Cabe resaltar que usan la escritura como proceso de aprendizaje para que los estudiantes se apropien más de los nuevos conocimientos, sin embargo, no existe una motivación que incentive la escritura de manera individual fuera del aula de clase.

\section{APRENDIZAJE}

Es importante recordar que para llegar a determinadas conclusiones sobre el aprendizaje de la escritura se realizaron un número significativo de encuestas a estudiantes de 
las carreras de Psicología, Licenciatura en Lengua Castellana, Licenciatura en Educación Artística y Cultural, Licenciatura en Educación Física, Recreación y Deporte y Licenciatura en Ciencias Sociales. En total fueron 258 encuestas conformadas por 6 preguntas cada una, de opción múltiple, con el fin de conocer cómo se han desarrollado los procesos de enseñanza y aprendizaje de la escritura en la Facultad de Educación de la Universidad de la Amazonia.

Sobre los tipos de textos, el $36 \%$ de los estudiantes encuestados expresan que escriben más ensayos, seguido del $18 \%$ quienes manifiestan que usan PowerPoint. La carrera que más utiliza los ensayos como tipo de texto durante sus materias es Ciencias sociales con un $16 \%$ de los estudiantes del programa. Los ensayos permiten a los estudiantes activarse académicamente, con el fin de mejorar su conocimiento, contrastarlo con el de otros autores y plasmarlos a través de la práctica escrita, por otra parte, el programa PowerPoint les permite a los estudiantes realizar presentaciones para que sus docentes les valoren sus trabajos. La riqueza de las mismas tipologías está en variar y encontrar la adecuada para cada situación, materia y carrera. "La tipología de texto no solo se justifica desde la diversidad de carreras profesionales en las que las experiencias se desarrollan, sino en las múltiples intenciones con las que se programan los cursos, talleres, electivas o pruebas" (González et al., 2015).

En cuanto a la metodología, los estudiantes perciben que se privilegia la explicación magistral, exposiciones y talleres en porcentajes similares, es decir que las dos primeras prácticas tienen un $32 \%$ y los talleres un $30 \%$. La metodología de tipo magistral consiste en la transmisión de información de docente - estudiante, lo que convierte al docente en la persona que dirige el aprendizaje siendo el emisor y el estudiante siendo el receptor. Aprender en la universidad no es un logro garantizado, depende de la interacción entre estudiantes y docentes (Carlino, 2005).

Los mecanismos de apoyo son usados por los docentes con el fin de enseñar las prácticas de la escritura, según los resultados de las encuestas un $65 \%$ de los estudiantes dicen que los mecanismos utilizados son la socialización de algunos ejemplos de los textos que solicitan escribir, seguido de la retroalimentación personalizada con un 19\%. La socialización resulta fundamental ya que a través del conocimiento de diferentes puntos de vista se amplía la visión, con los aportes de los demás.

Así mismo, el contexto que rodea al estudiante es muy importante para la realización de los textos e influye para que se den de mejor o peor manera, ya que por ejemplo si se encuentra en un lugar más tranquilo, sin estrés, la producción escrita va a ser diferente. Los lugares donde los estudiantes dicen realizar el proceso de escritura son dentro y fuera de clase, pero preferiblemente fuera con un $31 \%$ del total de encuestados, seguido por dentro y fuera de clase, pero preferiblemente en clase con un $29 \%$. Se puede notar con este resultado que los docentes dinamizan el proceso de escritura no centrándolo en un solo lugar sino variándolo.

En cuanto al propósito por el cual los estudiantes escriben, según las encuestas realizadas son en su mayoría por aprender en un $63 \%$ seguido por responder las exigencias del docente en un $21 \%$. Los criterios de evaluación, que son tenidos en cuenta por los docentes a la hora de calificar los escritos son principalmente el nivel de argumentación con un 30\%, además de la originalidad con un $18 \%$ y el manejo de las normas APA con un $16 \%$, como se puede comprobar en la Figura 2, sin embargo, los estudiantes del programa de Lengua Castellana dicen que se exige más en la originalidad de estos textos. 


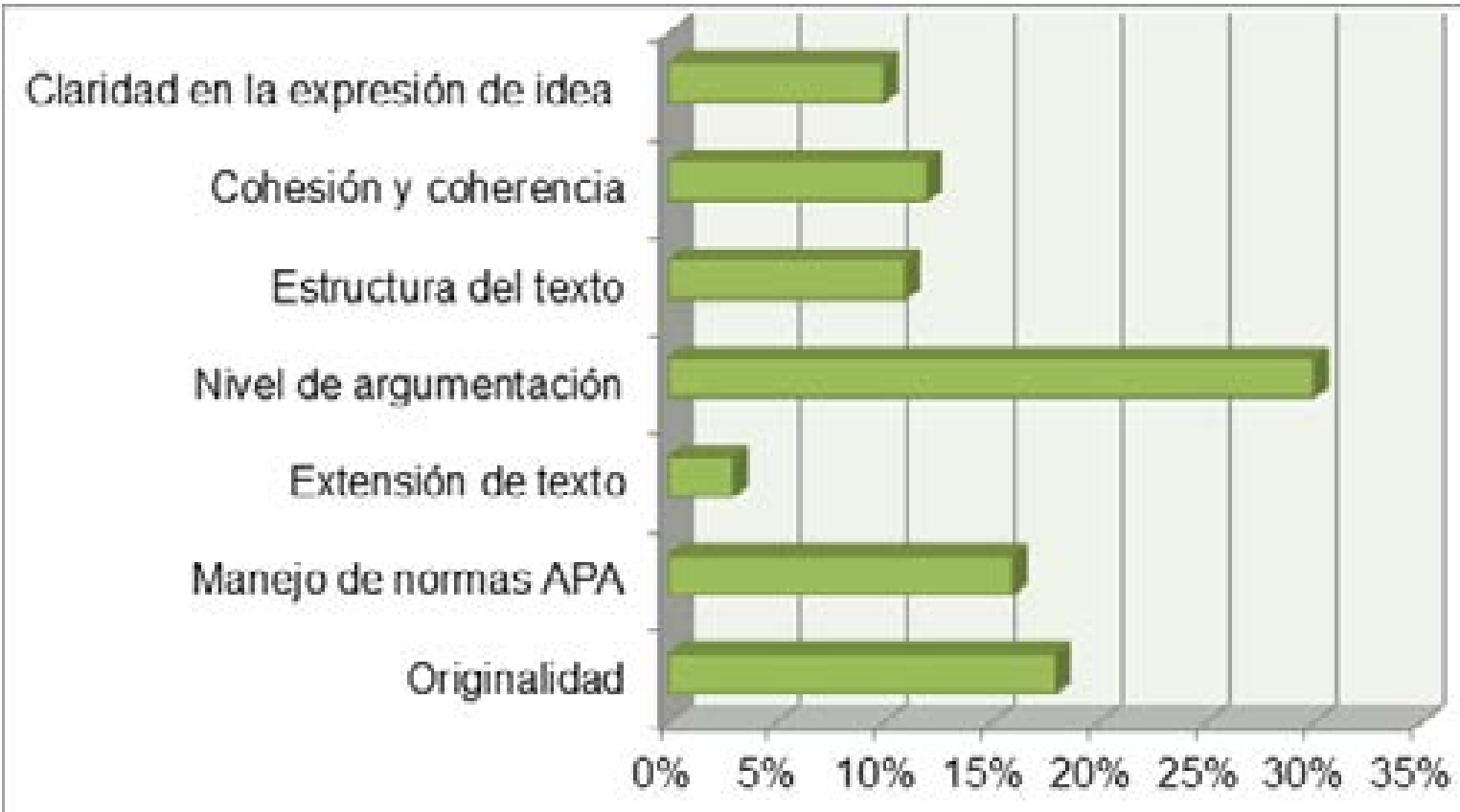

Figura 2: Criterios utilizados por los docentes para evaluar la producción escrita en la Facultad de Educación.

Finalmente, se puede afirmar que la escritura representa un pilar fundamental para la construcción del conocimiento académico de cada estudiante. El paso de los estudiantes por la universidad representa el camino de preparación profesional, por eso es importante pensar con rigurosidad su formación. Por lo general, la escritura se emplea para cumplir requerimientos y no como una construcción de aprendizaje, por ello es fundamental que se creen y ejecuten estrategias que permitan incentivar a los estudiantes de la educación superior a escribir, con el fin de ampliar sus conocimientos y reforzar los adquiridos.

\section{CONCLUSIONES}

A partir de la presente investigación surgen 3 grandes conclusiones, una con relación a cada categoría. Respecto a la primera categoría relacionada con política de la escritura, es importante resaltar que la escritura es un proceso fundamental en el ámbito académico; contribuye a la formación académica e intelectual, por ello es necesario que, en las instituciones educativas, especialmente en las de educación superior se fortalezcan los lineamientos de base para orientar la producción textual. En esta investigación se pudo evidenciar que la escritura no se estructura teniendo en cuenta las características propias de cada carrera o el ámbito donde se va a desempeñar el profesional en formación, ya que no hay lineamientos claros sobre la manera en la cual se debe enseñar la escritura y, por lo tanto, los docentes no tienen unos referentes concretos para orientar estos procesos.

Frente a la segunda categoría, enseñanza de la escritura, la mayoría de los docentes no tienen claros los lineamientos teórico - conceptuales y prácticos necesarios para la enseñanza de la escritura, es decir, aunque existan determinadas políticas para la enseñanza de la escritura, si estas no son socializadas ni apropiadas por los docentes, pierden su razón de ser. Los docentes tienen sus propias maneras para orientar el proceso de escritura con los estudiantes, como implementar un enfoque constructivista, enseñar a través de clases magistrales o 
talleres, solicitar ensayos e informes y evaluar a través de distintos métodos. Formas dispersas de enseñar que se confunde con el concepto de autonomía docente. En el mejor de los casos se transmite una idea de escritura epistémica, dejando un lado el potencial humanizador de la misma.

En cuanto al aprendizaje de la escritura, queda claro que muchos estudiantes escriben por escribir, como requisito para obtener notas. La producción escrita es orientada principalmente hacia la construcción de ensayos e informes y su diseño se orienta de forma magistral. Pero también se determinó que un número significativo de estudiantes han logrado comprender el potencial epistémico y humanizador de la misma.

Por último, es importante pensar las políticas de la escritura en coherencia con los procesos de enseñanza y aprendizaje de esta. También, verla de forma trasversal a todas las disciplinas de la universidad. Para la creación y/o actualización de las políticas de enseñanza y aprendizaje de la escritura se debe tener en cuenta la perspectiva de toda la comunidad educativa universitaria, es decir, tanto de docentes como de estudiantes, de tal manera que la universidad y, en este caso, la escritura, responda a unas determinadas necesidades socioculturales.

\section{REFERENCIAS BIBLIOGRÁFICAS}

Baeza Martín, R. (2012). Escribir y leer desde un enfoque constructivista. http://uvadoc.uva. es/handle/10324/1986

Carlino, P. (2005). Escribir, leer y aprender en la universidad Una introducción a la alfabetización académ ica (Fondo de Cultura Económica (ed.); Primera). https:// www.aacademica.org/paula.carlino/3.pdf

Carlino, P. (2009). Prácticas y representaciones de la escritura en la universidad: los casos de Australia, Canadá, EEUU y Argentina. Cuadernos de Psicopedagogía, 12,
6-17. https://www.aacademica.org/paula. carlino/189.pdf

Cassany, D. (1996). La cocina de la escritura. In Anagrama (Ed.), Anagrama. https:// bibliodarq.files.wordpress.com/2016/11/ cassany-d-la-cocina-de-la-escritura.pdf

Cassany, D., \& Morales, O. A. (2009). Leer y escribir en la universidad: los géneros científicos. In D. Cassany (Ed.), Para ser letrados. Voces y miradas sobre la lectura (1a ed., pp. 109-128). Paidós. https://www. researchgate.net/publication/301548145 Leer_y_escribir_en_la_universidad_los_ generos_cientificos

Colmenares, M., \& Delgado, F. (2008). La correlación entre rendimiento académico y motivación de logro: elementos para la discusión y reflexión. REDHECS: Revista Electrónica de Humanidades, Educación y Comunicación Social., 3(5), 179-191. https://dialnet.unirioja.es/servlet/ articulo?codigo $=2737310$

Flower, L., \& Hayes, J. R. (1996). La teoría de la redacción como proceso cognitivo. En Textos en contexto. Buenos Aires: Lectura y Vida, 1, 74-119. http://media.utp.edu. co/referencias-bibliograficas/uploads/ referencias/libro/518-flowers-y-hayes-lateoria-de-la-redaccion-como-procesocognitivopdf-xwyX0-articulo.pdf

Fragoso Fernández, E. (2006). Concepto de Política y Vida Cotidiana. Xihmai, 1. https://dialnet.unirioja.es/servlet/ articulo?codigo $=4953719$

Freire, P. (2011). Pedagogía Del Oprimido (Siglo XXI (ed.); 14th ed., Vol. 257, Issue November). https://doi.org/10.3163/15365050.98.2.021 
García Gajardo, F., Fonseca Grandón, G., \& Concha Gfell, L. (2015). Aprendizaje y rendimiento académico en educación superior: un estudio comparado. Actualidades Investigativas En Educación, 15 (3), 1-26. https://doi.org/http://dx.doi. org/10.15517/aie.v15i3.21072

Giraldo Giraldo, C. (2015). La escritura en el aula como instrumento de aprendizaje. Estudio en universidades. Ánfora, 22 (38), 39-59. https://www.redalyc.org/ pdf/3578/357839273002.pdf

González Pinzón, B. Y., Salazar-Sierra, A., Molina Ríos, J. A., \& Moya-Chaves, S. (2015). Acciones para la construcción de una política de lectura y escritura universitaria. Folios, 41, 143-155. http:// www.scielo.org.co/pdf/folios/n41/n41a10. pdf

González Pinzón, B. Y., \& Vega, V. (2014). Lectura y escritura en la educación superior colombiana: Herencia y deconstrucción. Interacción, 12, 195-201. https://doi. org/https://doi.org/10.18041/1657-7531/ interaccion.0.2325

Graham, S., Harris, K. R., \& Larsen, L. (2001). Prevención e intervención de las dificultades de escritura para estudiantes con dificultades de aprendizaje. Learning Disabilities Research and Practice, 16(2), 74-84. https://doi.org/10.1111/09388982.00009

Guelmes Valdés, E. L., \& Nieto Almeida, L. E. (2015). Algunas reflexiones sobre el enfoque mixto de la investigación pedagógica en el contexto cubano. Universidad y Sociedad, 7(2), 23-29. http:// scielo.sld.cu/pdf/rus/v7n1/rus03115.pdf

Martín-Barbero, J., \& Lluch, G. (2011). Proyecto: Lectura, escritura y desarrollo en la sociedad de la información (CERLALC (ed.); Primera). http://www.cerlalc.org/ Informe_Final_Lecura_Desarrollo.pdf

Mendoza, D. (2016). La Enseñanza De La Escritura En La Universidad: ¿Es Un Deber? Legenda, 20(23), 47-66. http:// erevistas.saber.ula.ve/index.php/legenda/ article/view/9091

Meneses Benítez, G. (2007). El proceso de enseñanza - aprendizaje: el acto didáctico [Universitat Rovira I Virgili]. https://www. tdx.cat/bitstream/handle/10803/8929/ Elprocesodeensenanza.pdf

Patiño Diaz, G. (2013). Escritura y universidad. Guía para el trabajo académico (Editorial Universidad del Rosario (ed.); Primera). https://ebook.urosario.edu.co/pdfreader/ escritura-y-universidad

Pérez Abril, M., \& Rincón Bonilla, G. (2013). ¿Para qué se lee y se escribe en la universidad colombiana? Un aporte a la consolidación de la cultura académica del país (Pontificia Universidad Javeriana (ed.); Primera). https://wac.colostate.edu/ books/colombian/highered.pdf

Roth Deubel, A.-N. (2002). Políticas públicas, formulación, implementación y evaluación (M. P. Jiménez Rodríguez (ed.); 1a ed.). Panamericana Formas e Impresos S.A. https://polpublicas.files.wordpress. com/2016/08/roth_andre-politicaspublicas-libro-completo.pdf

Sesento García, L. (2008). Modelo sistémico basado en competencias para instituciones educativas públicas. [Centro de Investigación y Desarrollo del Estado de Michoacán]. https://www.eumed.net/ tesis-doctorales/2012/lsg/index.htm 
Uribe Álvarez, G., \& Camargo Martínez, Z. (2011). Prácticas de lectura y escritura académicas en la universidad colombiana. Magis, Revista Internacional De Investigación En Educación, 3(6). https://revistas.javeriana. edu.co/index.php/MAGIS/article/view/3543

Vargas Franco, A. (2005). Escribir en la universidad: reflexiones sobre el proceso de composición escrita de textos académicos. Lenguaje, 33, 97-125. https://media.utp. edu.co/referencias-bibliograficas/uploads/ referencias/articulo/1209-escribir-en-launiversidad-reflexiones-sobre-el-procesode-composicion-escrita-de-textosacademicospdf-4KJ7j-articulo.pdf

Villalonga Gómez, C., \& Nuere Salgado, L. (2017). La renovación de la metodología en un contexto académico y su traslación educativa a la formación corporativa a través de los MOOC. La Cuestión Universitaria, 9, 136-152. http://polired. upm.es/index.php/lacuestionuniversitaria/ article/view/3573/3654

Zapata-Ros, M. (2015). Teorías y modelos sobre el aprendizaje en entornos conectados y ubicuos. Redalyc, 16(1), 69-102. https:// www.redalyc.org/pdf/5355/535554757006. pdf

Zhizhko, E.A. (2014). Laenseñanza dela escritura y lectura de textos académicos a los futuros investigadores educativos. Scielo, 14(65), 99-113. http://www.scielo.org.mx/ scielo.php?script=sci_arttext\&pid=S1665$26732014000200007 \&$ Ing=es\&tIng=es

Zuluaga Gómez, R. D. (2008). 300 estrategias de animación a la lectura (Ruben Darío Zuluaga Gómez (ed.); Primera). 